\title{
Laboratory environment monitoring and specimen transport robots
}

\author{
Yi Chang Wu$u^{1}$, Huan-Chun Wang ${ }^{2}$ \\ ${ }^{1}$ Department Forensic Science Division, Investigation Bureau, Ministry of Justice, Taiwan \\ ${ }^{2}$ Department of Electronic and Computer Engineer, National Taiwan University of Science and Technology, Taiwan
}

\begin{tabular}{l} 
Article Info \\
\hline Article history: \\
Received Jun 3, 2019 \\
Revised Aug 23, 2019 \\
Accepted Oct 6, 2019 \\
\hline
\end{tabular}

Keywords:

Monitoring laboratory, Robot

ROS

Temperature and humidity

sensors

\begin{abstract}
Robots have been used in various areas to replace manpower, reduce costs, and facilitate more effective resource allocation. This study sought to assist the business of the bureau by developing two robots using the Robot Operating System. The developed robots have autonomous intelligent navigation functions and are suited to monitor the environment of the laboratories in the bureau. One robot had a temperature and humidity sensor and an infrared thermal camera, and it could be used to patrol and monitor the laboratory environment. The other robot had drawers in which specimens could be placed; robotic arm in the elevator could coordinate and control elevators, enabling the robot to move and transport specimens autonomously. Plenty of tests were conducted to verify the feasibility and practicality.
\end{abstract}

Copyright () 2019 Institute of Advanced Engineering and Science. All rights reserved.

\section{Corresponding Author:}

Yi Chang $\mathrm{Wu}$,

Forensic Science Division,

Investigation Bureau, Ministry of Justice,

No. 74, Zhonghua Rd., Xindian Dist., New Taipei City 231, Taiwan (R.O.C.).

Email: shintenwu@gmail.com

\section{INTRODUCTION}

Thanks to technological maturity, experience accumulation, and product renewal, much progress has been made in the development of robots since the first robot was constructed. Robots can partially replace manpower and increase efficiency, and they are reliable and easily manageable. Applying robots to certain tasks has become increasingly common. G. Indrawan et al. aimed to preserve Balinese script and thus presented a robotic system including a transliterator web application and a robotic arm, which can be used to give Balinese script writing knowledge [1]. N. S. Ali et al. tried to avert hazardous tasks such as demining by designing a controllable computer robot to detect metals [2]. To help people with household chores, J. Lee et al. developed an Arduino-based multifunction floor cleaning robot that can both vacuum and wipe [3]. H. Widyantara $\mathrm{et} \mathrm{al}$. proposed an olfactory mobile robot integrating omnidirectional thermal anemometers and unmanned devices to trace harmful gas in open air [4]. E. Abana et al. developed a robotic rake to mix and turn paddy during sun drying, which is significant to maintain the quality of grains [5].

Among these applications, some of current research, products, and patents have primarily emphasized the use of robots for laboratory monitoring and object transport. For laboratory monitoring, previous studies have investigated integrating robots with monitoring cameras, sensors, and network functions. Some monitoring systems can even provide remote monitoring and alert functions concurrently alongside other functions. Abdul Aziz et al. used wireless sensors to monitor laboratory temperature during oil palm tissue culturing [6]. Joshi et al. displayed temperature on liquid crystal displays to reduce temperature fluctuations induced by people entering and exiting the IVF laboratory [7]. People are used to installing several cameras to continuously monitor environment, which generate a large amount of data. Liu 
et al. employed multiple sensors to determine the locations where accidents occur before switching on cameras, which contributed to energy conservation in comparison with conventional method [8]. A security monitoring system designed by Zhao et al. integrated cameras and sensors. Their monitoring server could display emergency alerts on a web page and signal any alerts using light and sound [9]. Jihua et al. transmitted images captured by a USB camera to a backup server, enabling users to view the images remotely and immediately, thereby achieving effective laboratory monitoring [10].

Research on robots used for transportation is relatively lacking, and thus we selected similar patents for analysis. Open patents for robots of this type mostly have basic functions such as autonomous mobility implemented by different methods. The main structure of "AUTONOMOUS MOBILE PICKING" [11] involves a vehicle base with an area for storing items and a mechanical arm. The base moves autonomously and can conduct item retrieval. The "Method and system for generating navigation data and transporting object" [12] is one object transport system. According to this system, the robot moves based on usergenerated navigation data. The elevator was operated by a separate robotic arm using an image recognition device that could press the elevator buttons, thereby allowing the robot to move around multilevel buildings.

As mentioned above, robots have been widely applied, whereas they are still uncommon in public sectors. This study demonstrated the creation of two robots which were constructed for laboratory monitoring and specimen transportation, and the hardware foundation for each robot we adopted were TurtleBot 3 with Lidar equipment. Through this study, besides the aim to utilize robots to gather and provide the environmental characteristics and assist the businesses of the bureau, simultaneously we sought to promote the use of smart technologies and ameliorate manpower shortage in the public sectors. It is worth mentioning that at present the laboratory monitoring robot patrol in the building after work everyday and the specimen transport robot also begin to serve in the building, driving the development of smart machinery in the bureau.

\section{RESEARCH METHOD}

This study developed several robotic technologies independently using the Robot Operating System (ROS) [13]. The ROS provides most of the functions of traditional operating systems such as hardware layer abstraction, low-level equipment control, inter-process message transmission, and package management. Additionally, relevant tools and procedural libraries are provided that can be used to acquire, compile, and edit code and achieve distributed computing. The ROS standard package provides various stable and adjustable robot algorithms. The standardized ROS communication interface means that developers can devote more time on design and actualization of new ideas and computations, thereby avoiding repetition of existing research outcomes. Modern robots usually require multiple computers to calculate the numerous processes they conduct. Thus, a robot can be equipped with several computers, with each computer powering a part of the robot's transducer and driver. Alternatively, users can send control commands to a robot through their computers, such as a tablet or smartphone. This type of human-machine interactive interface can be considered as part of a distributed system. Therefore, the ROS can help resolve communication problems that arise between different processes when several computers are part of a distributed system.

Based on the ROS, we developed functions such as autonomous smart navigation, a human-machine remote control interface, fire and flood alerts/environmental temperature and humidity monitoring, flexible item storage and transport, and elevator operation; the design of each function was as follows:

\subsection{Autonomous smart navigation}

\subsubsection{Mapping}

High-Precision Lidar as shown in Figure 1 was used to construct a customized map as shown in Figure 2 of the building using the gmapping algorithm [14]. The Rao-Blackwellized particle filter was used with the gmapping algorithm to achieve simultaneous localization and mapping (SLAM). A study [15] indicated that gmapping has high stability and excellent performance in terms of the error rate and CPU load.

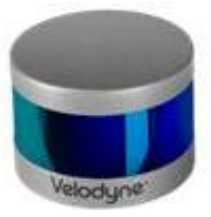

Figure 1. Lidar unit 


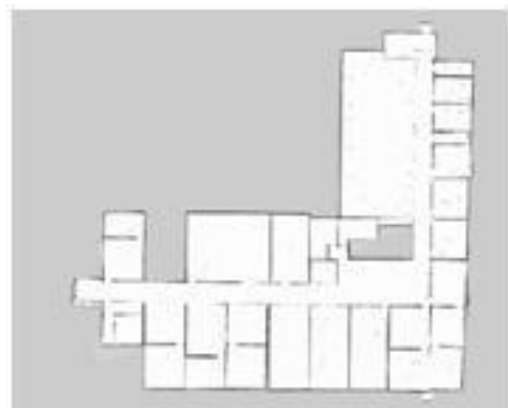

Figure 2. Customized map of the building

\subsubsection{Positioning}

Taking the data from the Lidar and an inertial measurement unit as shown in Figure 3, the adaptive Monte Carlo localization (AMCL) algorithm [16] was adopted to achieve positioning as shown in Figure 4. The customized map was used with the algorithm to dynamically construct probability distributions of particles. Then, the Lidar-measured values were used to adjust the probability distributions until the positioning results converged.

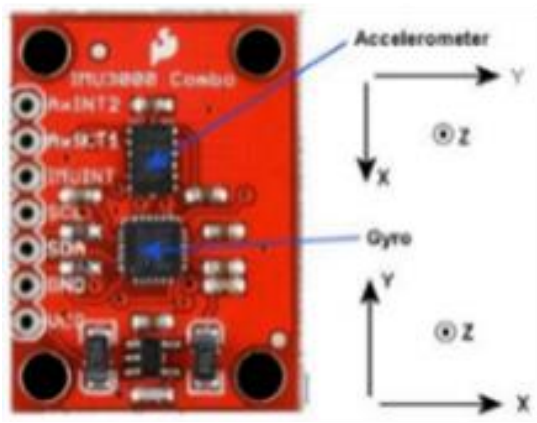

Figure 3. Inertial measurement unit

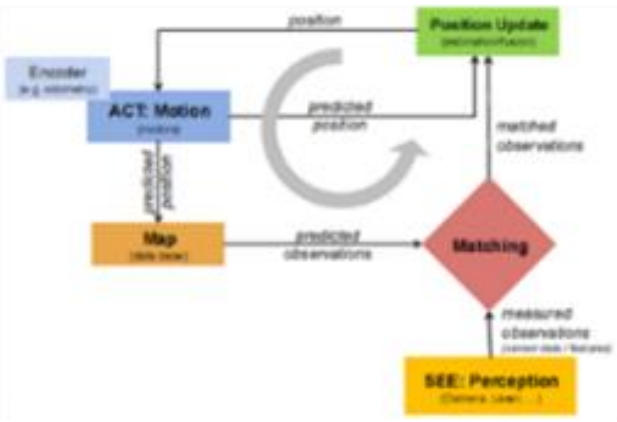

Figure 4. AMCL positioning

\subsubsection{Route planning and following}

The probabilistic roadmap (PRM) algorithm [17] was used for route planning by constructing connections between nodes that were subsequently used to locate obstacle-free routes between the starting and finishing point as shown in Figure 5. The Pure Pursuit algorithm [18] was used to execute the planned route, and look-ahead points were adjusted to ensure the route was smoothly and correctly taken as shown in Figure 6. 


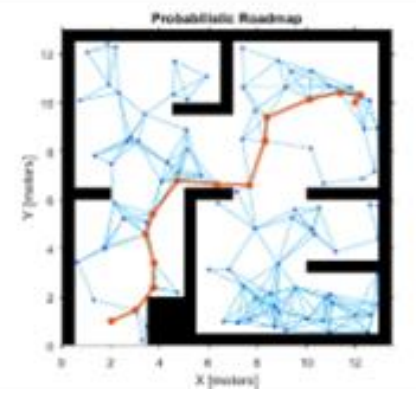

Figure 5. PRM route planning

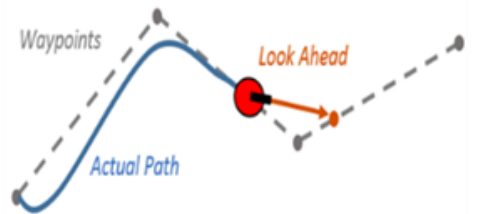

Figure 6. Pure Pursuit route execution

\subsubsection{Dynamic environment detection and obstacle avoidance}

The vector field histogram (VFH+) algorithm [19, 20] was adopted for dynamic environment detection and obstacle avoidance as shown in Figure 6. This algorithm used the data received from the sonar as shown in Figure 7 and Lidar as shown in Figure 1 to construct the polar histogram of obstacles. Subsequently, the histogram thresholds and minimum turning radium were used to determine the required route for obstacle avoidance as shown in Figure 8.

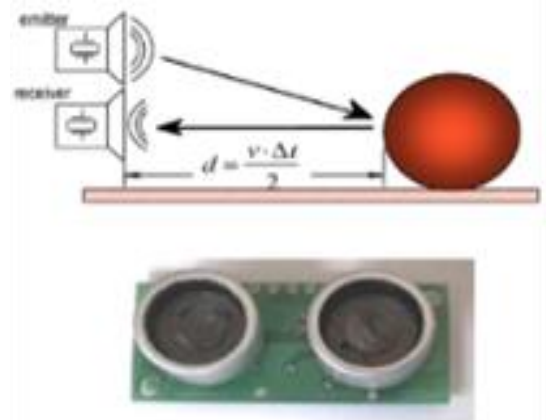

Figure 7. Sonar elements

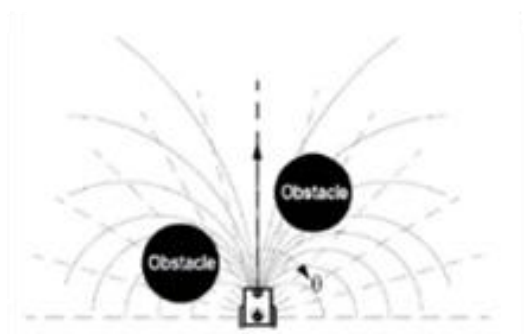

Figure 8. Dynamic environment detection and obstacle avoidance 


\subsection{Remote human-machine control interface}

The Representational state transfer (RESTful) API [21, 22] as shown in Figure 9 not only enabled us to operate intelligent machines on websites, applications, and mobile devices, but also sent images from its visual system to users as shown in Figure 10.

The RESTful API comprises three elements [23]:

- $\quad$ A URL for the web service, (e.g., http://example.com/resources/).

- A data-interchange format that is accepted and returned by the web service, (e.g., JSON).

- RESTful methods for making requests that are supported by the web service, (e.g., POST, GET, PUT, or DELETE).

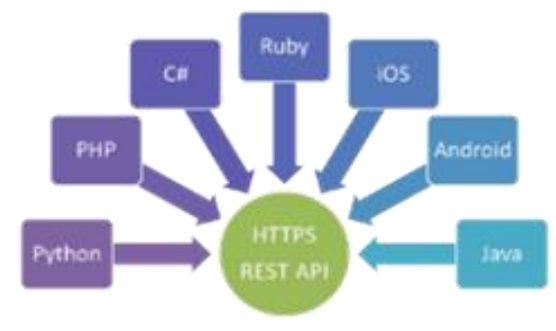

Figure 9. RESTful API framework

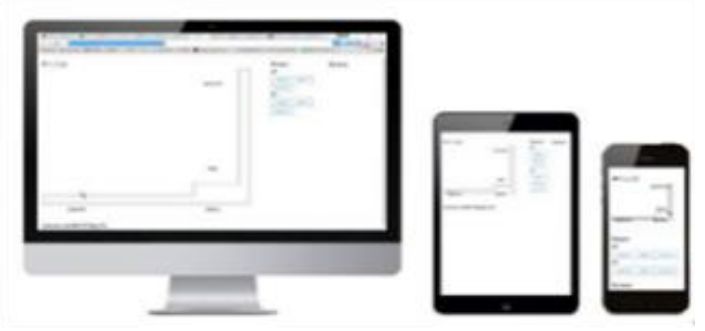

Figure 10. Human-machine remote control interface (exp: mobile devices such as tablets, smart phones...etc.)

The RESTful API uses HTTP as the underlying protocol $[22,24]$. Compared with conventional web services, RESTful is lightweight with both client and server sides. On the client side, HTTP is used to request resources from the server side. The server side is responsible for processing requests and allocating resources. HTTP operation that can be used on websites, applications, and mobile devices enables quick and simple operation of smart machines using a visual interface.

\subsection{Fire and flood alerts/environmental temperature and humidity monitoring}

Environmental changes can occur anywhere within an area, and therefore in this study, the Optris PI 230 infrared thermal camera as shown in Figure 11 and DHT22 temperature and humidity sensor were selected to conduct environmental monitoring and provide early warning of potential fire and flood conditions. The purpose of these sensors was to compensate for the inadequacy of traditional fire and flood alerts by quickly detecting changes in the environment. The Optris PI 230 infrared thermal camera enabled environmental temperature monitoring. By examining temperature distributions, the camera could monitor for fire and flood conditions; moreover, it provided a convenient means for human-machine remote operation through client-side viewing of the environment's temperature. The DHT22 temperature and humidity sensor was used with an Arduino UNO board to record the environmental temperature and humidity, thereby also enabling early warning and prevention of hazardous environmental conditions. 


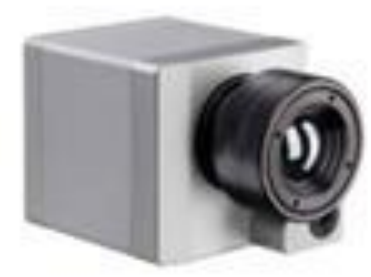

Figure 11. Optris PI 230 infrared thermal camera

\subsection{Flexible item storage and transport}

The developed transport robot integrated item-carrying and autonomous navigation functions. The base of the robot provided the storage areas for users to placed and classified items. Using the central monitoring system designed in MATLAB, a target location for item delivery was selected. Subsequently, the robot traveled to the designated location and notified the recipient to complete specimen transport. The Arduino UNO board commanded an actuator to open and close the storage areas and sent instructions to the Sabertooth motor controller to drive the motor to rotate the left and right wheels.

\subsection{Elevator operation}

After the robot arrived at the designated position, it transmitted a signal over WiFi to the robotic arm in the elevator to press the required buttons to operate the elevator as shown in Figure 12. The highly stable vertical articulated robotic arm was connected to a control board using a USB port. It was placed in a fixed position above the elevator buttons, and the angle and force of the button pressing were set in advance. When users selected a specific floor using the central control system, the robotic arm pressed the buttons according to the command. This enabled the other robots to move freely around the building independently.

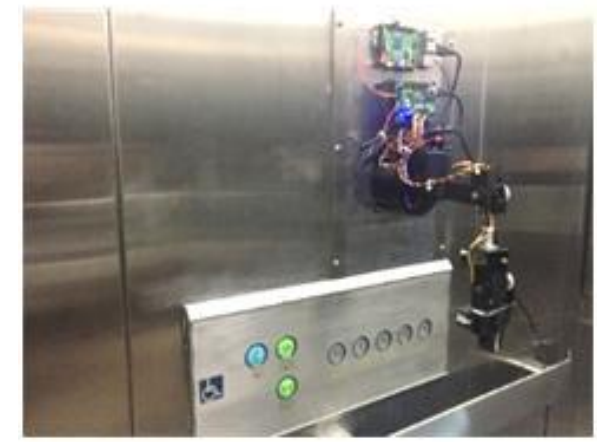

Figure 12. Button-pressing robotic arm in elevator

\section{RESULTS}

\subsection{Laboratory monitoring robot}

\subsubsection{Composition}

The laboratory of monitoring robot consist of a Lidar unit, a thermal camera, a temperature and humidity sensor, microprocessors, ROS, and peripheral equipment. The laboratory of monitoring robot is shown in Figure 13. 


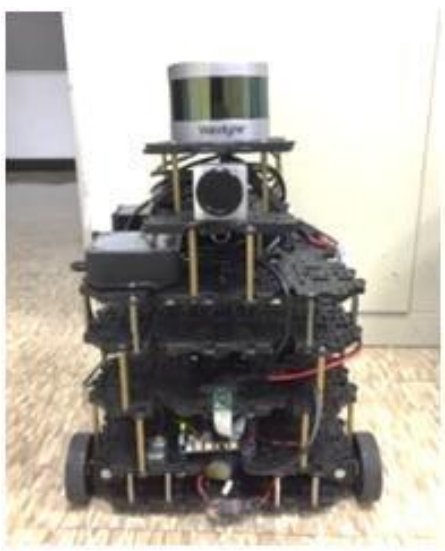

Figure 13. External appearance of the laboratory monitoring robot

\subsubsection{Laboratory monitoring robot user interface}

Figure 14 shows the central monitoring system of the laboratory monitoring robot. The explanations of the interfaces are:

(1) Map displaying current location and current floor.

(2) Robot's current floor.

(3) Two speedometers on the left and right display current linear velocity and angular velocity, respectively.

(4) Selection of floor for the robotic arm in the elevator.

(5) Alarm: When fire, high temperature, flood, low temperature, and foreign objects are detected, an alarm sounds and an email is sent to the user.

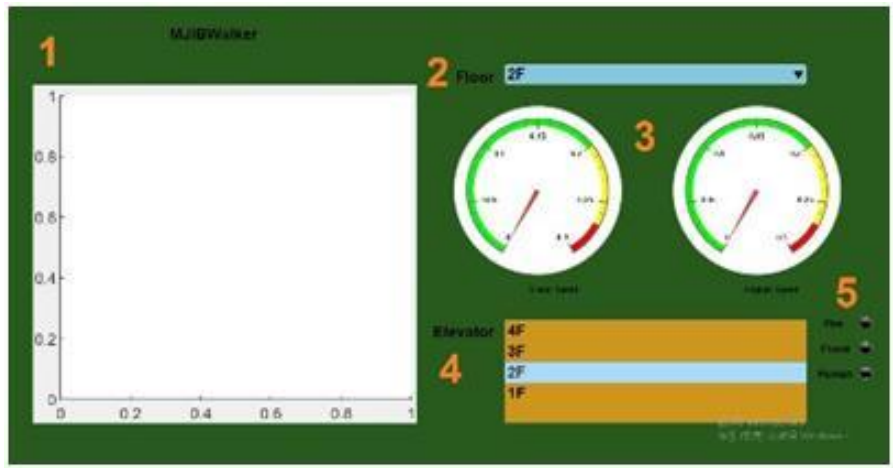

Figure 14. Central monitoring system of the laboratory monitoring robot

\subsubsection{Testing}

The laboratory monitoring robot was used to patrol a building as shown in Figure 15. When the robot detected abnormal conditions such as high or low temperature as shown in Figure 16 or a foreign object as shown in Figure 17, it produced an alert as shown in Figure 18 and sent an email to notify the users. 

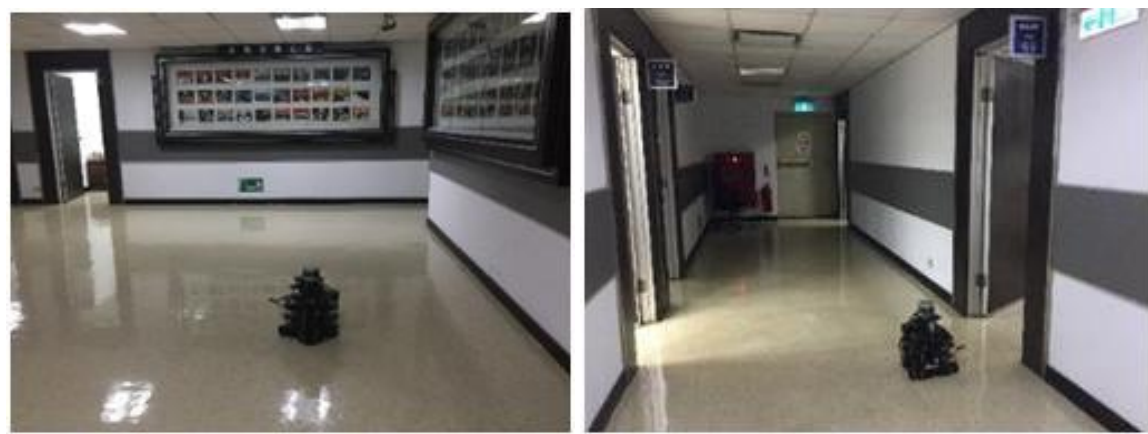

Figure 15. Laboratory monitoring robot on patrol

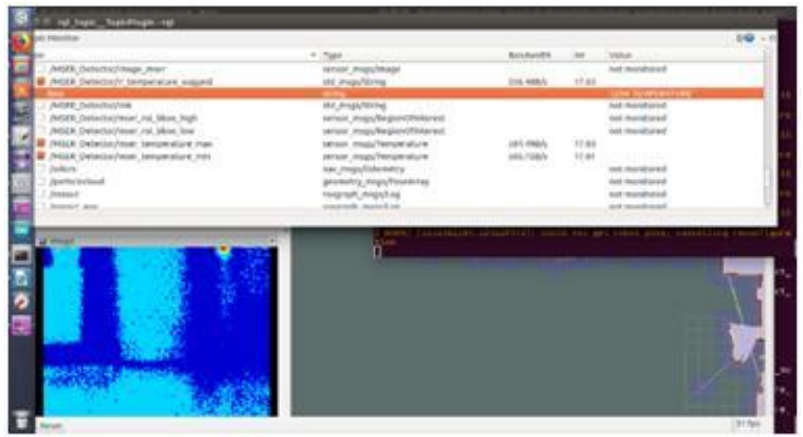

Figure 16. Low temperature detection

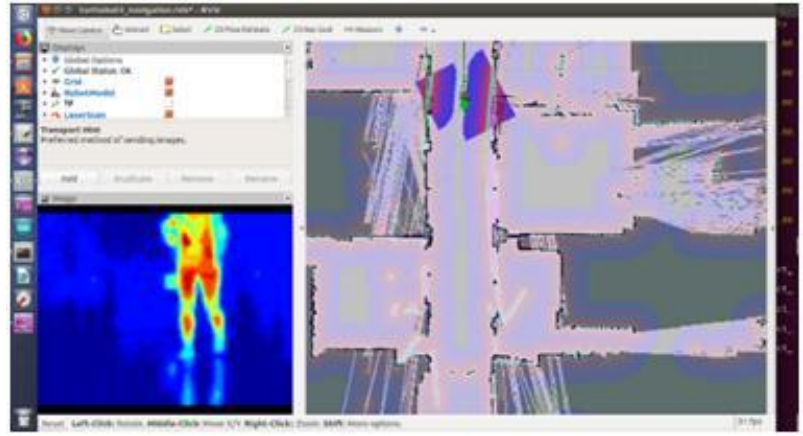

Figure 17. Foreign object (human body) detection

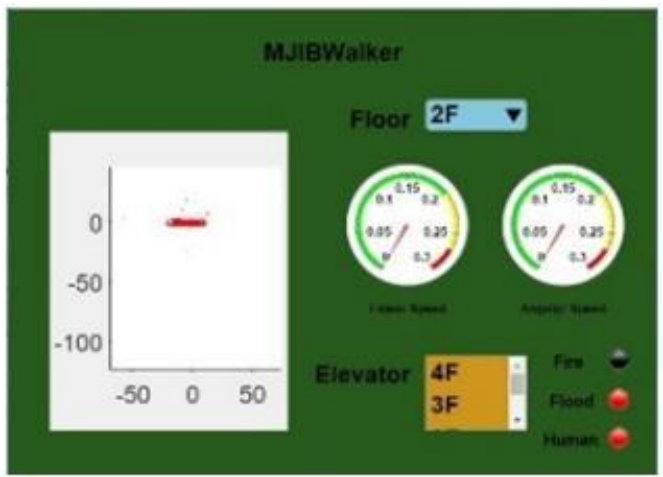

Figure 18. Alert display on user interface 


\subsubsection{Comparison}

We compared the laboratory monitoring robot and reference researches; the details and analysis are shown in Table 1.

Table 1. Comparison between laboratory monitoring robot and reference researches

\begin{tabular}{|c|c|c|c|}
\hline Item & Laboratory monitoring robot & Reference researches & Analysis \\
\hline Sensor & Humidity and temperature & Temperature & $\begin{array}{l}\text { Other than temperature, humidity is also a key } \\
\text { ambient factor in identification laboratories. } \\
\text { For example, water leakage can be detected } \\
\text { through humidity sensors. }\end{array}$ \\
\hline Mobility & Mobile and flexible & Fixed & $\begin{array}{l}\text { Fixed-type monitoring devices are convenient } \\
\text { for location confirmation but lack flexibility; } \\
\text { mobile monitoring robot enables specific areas } \\
\text { to be strengthened. }\end{array}$ \\
\hline Camera & Infrared thermal camera & Camcorder & $\begin{array}{l}\text { Infrared thermal makes it easy to identify heat } \\
\text { source and human body. }\end{array}$ \\
\hline Monitoring Area & Extensive & Constant & $\begin{array}{l}\text { Thanks to its mobility the monitoring robot is } \\
\text { able to get anywhere if the Internet is } \\
\text { connected. }\end{array}$ \\
\hline Power & Auto-ducking & Wired charging & $\begin{array}{l}\text { While wired charging is steady, the laboratory } \\
\text { monitoring robot will send a voice alert before } \\
\text { power exhaustion, and it contains an auto- } \\
\text { ducking function. }\end{array}$ \\
\hline
\end{tabular}

\subsection{Specimen transport robot}

\subsubsection{Composition}

The specimen transport robot consists of body with storage drawers, a computer, Lidar unit, a motor, and peripheral equipment. External appearance of the specimen transport robot as shown in Figure 19.

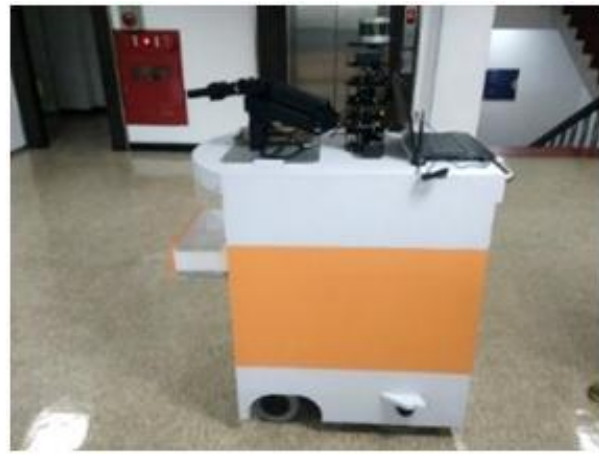

Figure 19. External appearance of the specimen transport robot

\subsubsection{Specimen transport robot monitoring system user interface}

Central monitoring system of the specimen transport robot is shown in Figure 20. The Explanation of the interface:

(1) Map displaying current location and current floor.

(2) Room the robot will travel to.

(3) Two speedometers on the left and right display current linear velocity and angular velocity, respectively.

(4) Switches for storage areas: the storage areas comprised three drawers that were controlled using an actuator. 


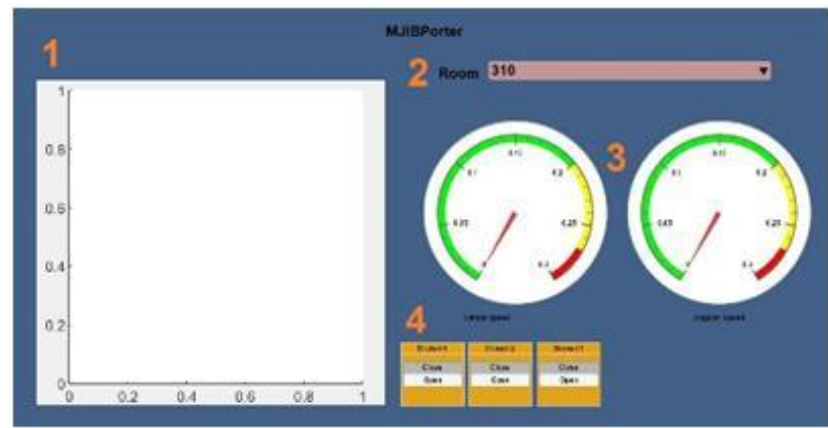

Figure 20. Central monitoring system of the specimen transport robot

\subsubsection{Testing} Figure 26

The process of the specimen transportation conducted by the robot was shown in Figure 21 to

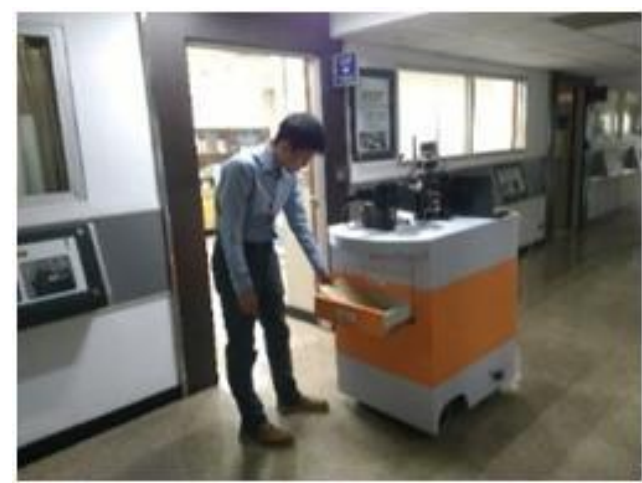

Figure 21. Opening a drawer through the central monitoring system (Figure 20), placing a sample, and selecting the target office

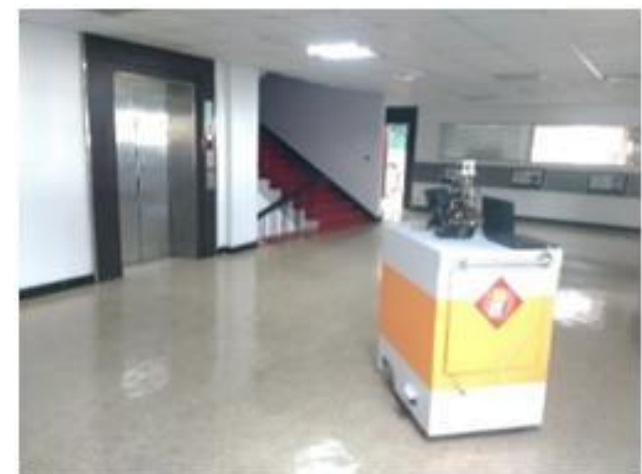

Figure 22. Robot moving to the elevator and sending a signal to the robotic arm in the elevator to open the elevator door 


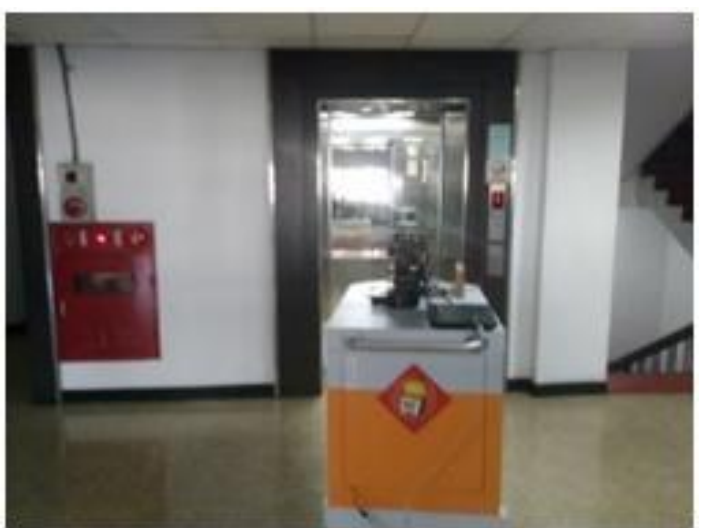

Figure 23. Robot entering the elevator

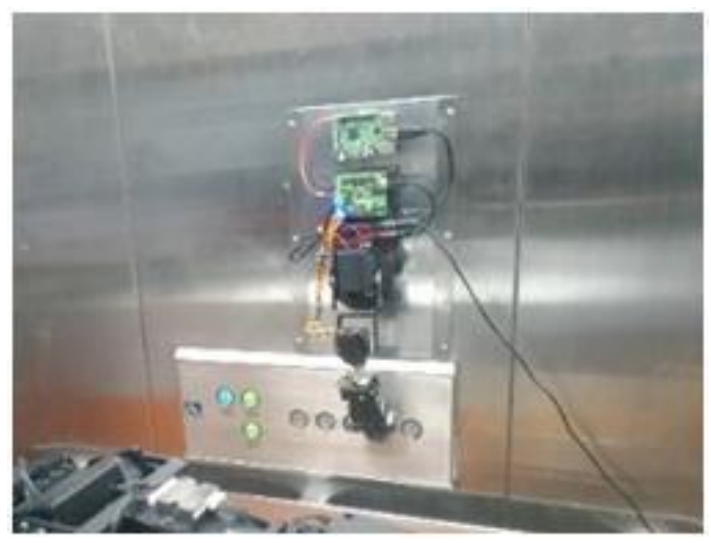

Figure 24. Robotic arm pressing the button for the floor on which the target office is

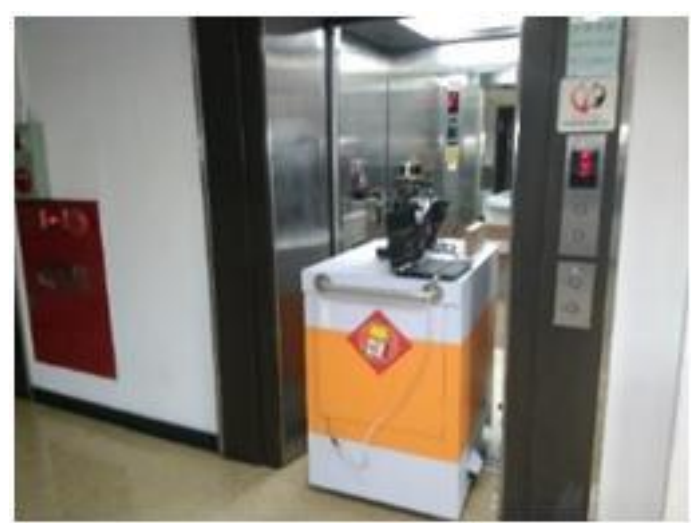

Figure 25. Robot exiting the elevator when the elevator reaches the target floor 


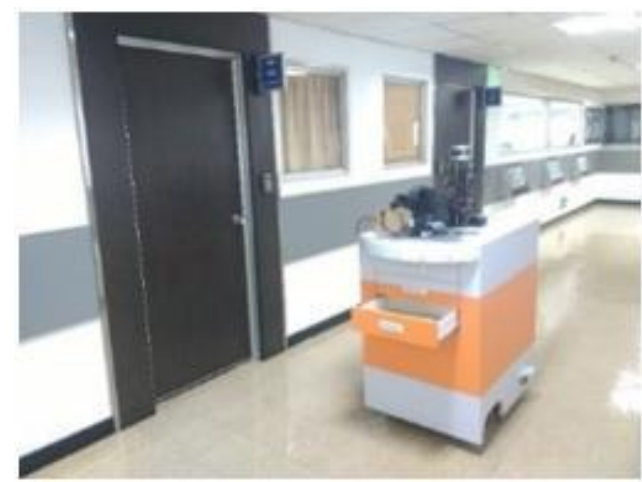

Figure 26. Robot arriving at the target office and opening the storage drawer

\subsubsection{Comparison}

The difference before and after using the specimen transport robot is shown in Table 2.

Table 2. Comparison between before and after using the specimen transport robot

\begin{tabular}{|c|c|}
\hline Before & After \\
\hline $\begin{array}{l}\text { The receipt and forwarding clerks need to ambulate in the } \\
\text { building to deliver specimens and official documents, urgent } \\
\text { or standard, to identification laboratories. The case officers } \\
\text { also have to return identified specimens and submit official } \\
\text { documents to upper supervisors according to the hierarchy } \\
\text { afterwards. }\end{array}$ & $\begin{array}{l}\text { With the assistance of the specimen transport robot, the clerks } \\
\text { can deal with urgent ones first; standard documents which are in } \\
\text { the majority can be collected and transported later by the robot. } \\
\text { All the case officers need to do is to place the specimens or } \\
\text { documents in the drawer and click on the monitoring system, } \\
\text { and then they can go back to work. Moreover, the robot is able }\end{array}$ \\
\hline $\begin{array}{l}\text { But it takes much time for the clerks and case officers to walk } \\
\text { around offices and they can only carry a few specimens and }\end{array}$ & $\begin{array}{l}\text { to carry a large number of objects at one time, thus sharing } \\
\text { responsibilities for objects delivery and saving time. }\end{array}$ \\
\hline
\end{tabular}

\section{CONCLUSION}

We have presented two robots, the laboratory monitoring robot and the specimen transport robot, which are the very first developed and applied in the bureau. Both have been tested for feasibility and practicality; the effectiveness of using smart robots to maintain environmental safety, assist business execution, ease the manpower shortage and improve resource allocation were also verified. So far the laboratory monitoring robot has performed its tasks for several months; also the specimen help transport dozens of objects every day. More importantly, employing self-developed and assembled robots can considerably reduce costs. In the future, the research attempt is to develop technologies such as facial and license plate recognition for use with robots; deep learning is to be employed to expand the robots' monitoring ranges and intensify park security.

\section{REFERENCES}

[1] G. Indrawan, et al., "LBtrans-Bot: A Latin-to-Balinese Script Transliteration Robotic System based on Noto Sans Balinese Font," Indonesian Journal of Electrical Engineering and Computer Science, vol. 12, no. 3, pp. 1247-1256, Dec 2018.

[2] N. S. Ali, et al., "Multi-Function Intelligent Robotic in Metals Detection Applications," TELKOMNIKA, vol. 17, no. 4, pp. 2058-2069, Aug 2019.

[3] J. Lee, et al., "Autonomous Multi-Function Floor Cleaning Robot with Zig Zag Algorithm," Indonesian Journal of Electrical Engineering and Computer Science, vol. 15, no. 2, pp. 1653-1663, Aug 2019.

[4] H. Widyantara, et al., "Wind Direction Sensor based on Thermal Anemometer for Olfactory Mobile Robot," Indonesian Journal of Electrical Engineering and Computer Science, vol. 13, no. 2, pp. 475-484, Feb 2019.

[5] E. Abana, et al., "Rakebot: a Robotic Rake for Mixing Paddy in Sun Drying," Indonesian Journal of Electrical Engineering and Computer Science, vol. 14, no. 3, pp. 1165-1170, Jun 2019.

[6] N. H. A. Aziz, et al., "Wireless System for Temperature Monitoring in Oil Palm Bio-laboratory," in 2009 Fifth International Conference on MEMS NANO, and Smart Systems, pp. 234-238, 2009.

[7] M. A. Joshi, et al., "Distributed Temperature Monitoring System for IVF Laboratory," in 2011 3rd International Conference on Electronics Computer Technology (ICECT 2011), pp. 282-284, 2011. 
[8] Chung-Hsin Liu, et al., "The Safety Design of the Laboratory Monitoring System," in The 6th International Conference on Networked Computing and Advanced Information Management (NCM 2010), pp. 509-513, 2010.

[9] G. Zhao, et al., "An Embedded Laboratory Security Monitoring System," in 2011 Third International Conference on Measuring Technology and Mechatronics Automation (ICMTMA 2011), pp. 395-398, 2011.

[10] Ye Jihua, et al., "Implementation of a Laboratory Video Monitoring System," in 2014 9th International Conference on Computer Science \& Education (ICCSE 2014), 2014, pp. 1028-1031.

[11] M. S. Khodl, D. M. Berghorn, and T. S. Hunter. (2018). United States Patent No. 0141755. Washington, DC: U.S. Patent and Trademark Office.

[12] Cheng-Xing Zhong, Peng Chen, and Jun-Po Chen. (2018). Taiwan Patent Publication No. 201816363. Taipei: Intellectual Property Office, MOEA.

[13] ROS.org [Internet]. Mountain View: Open Resource Robotics Foundation; [cited Apr. 17 2018]. ROS/Introduction. Available from: http://wiki.ros.org/ROS/Introduction.

[14] Giorgio Grisetti, et al., "Improved Techniques for Grid Mapping with Rao-Blackwellized Particle Filters," IEEE Transactions on Robotics, vol. 23, no. 1, pp. 34-46, Feb 2007.

[15] J. M. Santos, et al., "An Evaluation of 2D SLAM Techniques Available in Robot Operating System," in 2013 IEEE International Symposium on Safety, Security, and Rescue Robotics (SSRR 2013), pp. 1-6, 2013.

[16] D. Fox. "KLD-sampling: Adaptive Particle Filters," in NIPS'01 Proceedings of the 14th International Conference on Neural Information Processing Systems: Natural and Synthetic, pp. 713-720, 2011.

[17] L. E. Kavraki, et al., "Probabilistic Roadmaps for Path Planning in High-Dimensional Configuration Spaces," IEEE Transactions on Robotics and Automation, vol. 12, no. 4, pp. 566-580, Aug 1996.

[18] R. C. Coulter, "Implementation of the Pure Pursuit Path Tracking Algorithm," Technical Report CMU-RI-TR-9201, Robotics Institute, Carnegie Mellon University, Pittsburgh, PA, Jan. 1992.

[19] J. Borenstein, et al., "The Vector Field Histogram-Fast Obstacle Avoidance for Mobile Robots," IEEE Journal of Robotics and Automation, vol. 7, no. 3, pp.278-88, June 1991.

[20] I. Ulrich, et al., "VFH+: Reliable Obstacle Avoidance for Fast Mobile Robots," Proceedings. 1998 IEEE International Conference on Robotics and Automation, pp. 1572-1577, 1998.

[21] C. Pautasso, "RESTful Web Service Composition with BPEL for REST," Data \& Knowledge Engineering, vol. 68, no. 9, pp. 851-866, September 2009.

[22] P. Belimpasakis, et al., "A Platform for Proving Family Oriented RESTful Services Hosted at Home," IEEE Transactions on Consumer Electronics, vol. 55, no. 2, pp. 690-698, May 2009.

[23] Guru99.com [Internet]. Guru99. [cited May 17 2019]. RESTful Web Services Tutorial with Example. Available from: https://www.guru99.com/restful-web-services.html.

[24] C. Pautasso, et al., "Push-Enabling RESTful Business Processes," Internationl Conference on Service-Oriented Computing, vol. 7084, pp. 32-46, 2011. 


\section{BIOGRAPHIES OF AUTHORS}

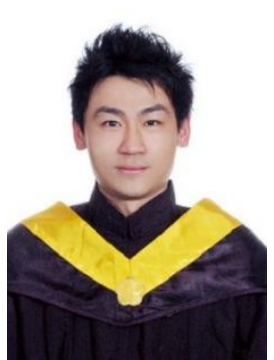

Yi Chang Wu

Yi-Chang Wu works for Investigation Bureau, Ministry of Justice. He is also a Ph.D. candidate of Electronic and Computer Engineering at National Taiwan University of Science and Technology.

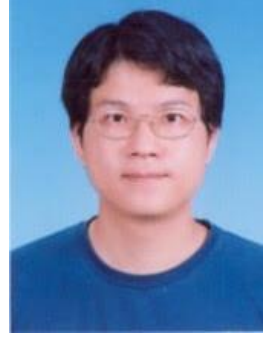

Huan-Chun Wang

Huan-Chun Wang received the MS and $\mathrm{PhD}$ degrees in Electrical Engineering from National

Chung Cheng University, Taiwan, in 1994 and 1999, respectively. During 2000-2004, he stayed in ITRI, where he was mainly involved in the project of WCDMA. Since 2004, he has been with the Department of Electronic Engineering of National Taiwan University of Science and Technology, Taiwan. His current research interests include iterative detection and MIMO systems.v 